\title{
GESTAÇÃO E DROGADIÇÃO: O IMPACTO DO ESTIGMA NO ATENDIMENTO PRÉ-NATAL
}

\section{Aline Oliveira da Silva1; Taís Regina Radiske ${ }^{2}$; Simoni Machado Santini ${ }^{3}$; Franciéli da Costa Rubert ${ }^{4}$; Patrícia Cristiane da Costa Dutra ${ }^{5}$ Félix Miguel Nascimento Guazina ${ }^{6}$}

\section{RESUMO}

Trata-se de uma revisão bibliográfica sobre a temática do uso de drogas na gestação. Por meio deste, procura-se apresentar uma reflexão sobre o atendimento pré-natal da gestante dependente química. Realizou-se revisão bibliográfica em artigos, pesquisas e normas técnicas que abordam o tema da gestação e do uso de drogas. O estudo reflete sobre o estigma presente no atendimento a esta demanda pelas equipes de saúde. Conclui-se que em grande parte, essas mulheres não acessam os serviços de saúde, não realizam o pré-natal e chegam aos hospitais somente na hora do parto, além de sofrerem preconceitos e serem estigmatizadas.

Palavras-chave: Drogas; Gestação; Integralidade do Cuidado.

Eixo Temático: Atenção Integral e Promoção à Saúde.

\section{INTRODUÇÃO}

O uso de drogas ilícitas é um problema social e de saúde de âmbito mundial que assola pessoas de diferentes classes sociais, etnias, idade e escolaridade. Quando se relaciona o uso de drogas ao gênero, o crescimento do número de mulheres que apresentam dependência química é significativo. O público feminino está mais vulnerável às doenças e aos agravos provocados pelo uso abusivo de

\footnotetext{
1 Assistente Social - Residente em Saúde Mental - UFN. Lineoliveiradasilva@hotmail.com

2 Psicóloga - Residente em Saúde Mental - UFN. Tais radiske@hotmail.com

3 Terapeuta Ocupacional - Residente em Saúde Mental - UFN. Santinisimoni@gmail.com

${ }^{4}$ Farmacêutica - Residente em Saúde Mental - UFN. Francielerubert7@gmail.com

${ }^{5}$ Enfermeira - Preceptora do Programa de Residência da UFN. Patycristiane@gmail.com

${ }^{6}$ Psicólogo - Doutor em Psicologia - UFN. Guazina@gmail.com
} 
EDUCAÇÃO, SAÚDE

ETECNOLOGIA

26 A 28 DE OUTUBRO DE 2021

drogas ilícitas, devido a busca por atendimento de saúde ocorrer de forma indireta (CARLINI, 2006).

Este aumento do número de mulheres com dependência química tem recebido atenção insuficiente e apresenta uma nuance muito particular que precisa ser observada: o estereótipo construído socialmente da mulher gestante usuária de drogas. Essas se encontram entre as mais vulneráveis, pois ficam expostas à prostituição, à exploração sexual, às infecções sexualmente transmissíveis (IST'S) e às gestações indesejáveis. Por isso, avaliar os efeitos do uso de drogas diretamente sobre a gestação é complexo, uma vez que precisam ser consideradas variáveis sociais, econômicas, geográficas, doenças pregressas, entre outras (BRASIL, 2012).

Os estudos relacionados ao tema têm apontado que o uso de crack, por exemplo, em mulheres gestantes, constitui-se em um grave problema de saúde pública, visto que pode acarretar várias consequências fisiológicas e sociais. Para as mulheres, pode causar, hipertensão, abortamentos, parto prematuro, morte fetal, dentre outras. Para os nascituros, pode causar irritabilidade, tremores, baixo peso ao nascer, além de consequências sociais para ambos, como abortamento ilegal, abandono do bebê, perda e destituição do poder familiar (MAIA; PEREIRA; MENEZES, 2015).

Ressalta-se que, entre as atribuições da atenção básica, está a captação e busca ativa das gestantes com esta problemática, tendo em vista seu acompanhamento na assistência ao pré-natal, uma vez que compete a este serviço de saúde a realização da estratificação de risco, a realização de visitas domiciliares, acolhimento e encaminhamento aos serviços de pré-natal de alto risco, caso seja necessário (BRASIL, 2013).

Diante desta realidade, é necessário que essas mulheres sejam acompanhadas de forma diferenciada durante toda a gestação, para que realizem um pré-natal de qualidade e exames para o diagnóstico de doenças e tratamentos. No entanto, observa-se uma realidade diferente. As gestantes não estão buscando atendimento na rede de atenção primária, não estão realizando o pré-natal adequado e chegam aos hospitais somente no momento do parto (MAIA; PEREIRA; MENEZES, 2015). 
Mediante o cenário exposto, este trabalho realiza uma revisão de literatura acerca dos autores que abordam o problema da drogadição em mulheres gestantes. Ainda, busca refletir sobre o atendimento dos serviços de saúde às mulheres gestantes dependentes químicas na realização do seu pré-natal, trazendo para o debate os fatores que dificultam o vínculo e permanência no acompanhamento.

\section{METODOLOGIA}

Trata-se de uma revisão de literatura em artigos, pesquisas e normas técnicas do Ministério da Saúde sobre a temática da gestação e do uso de drogas, apresentando reflexões sobre o estigma enfrentado pelas mulheres gestantes durante o atendimento pré-natal (CAMARGO et al; 2018).

\section{RESULTADOS E DISCUSSÕES}

\subsection{O ESTIGMA PRESENTE NO ACOMPANHAMENTO PRÉ-NATAL DE GESTANTES DEPENDENTES QUÍMICAS}

O uso de drogas por gestantes e puérperas é um problema de saúde pública complexo, haja vista que estas mulheres tendem a não aderirem à assistência ao prénatal, não comparecem aos serviços de saúde com regularidade, buscando-os apenas em casos de intercorrências mais graves (PORTO et al., 2018). Estudos demonstram que a não procura pelos serviços de saúde ocorre muitas vezes pelo preconceito por parte de quem oferta o cuidado (KASSADA et al., 2013, p. 470; BRASIL, 2012; CAMARGO et al, 2018).

Estas mulheres, até a realização do parto, muitas vezes não têm acesso a rede psicossocial, encontram-se com laços familiares rompidos ou fragilizados e não acessam os serviços de saúde. É uma situação complexa, mas que muitas vezes é abordada pelo viés do desvio, do caráter incapacitante e moralizante pelas equipes de saúde (BRITES, 2015; CAMARGO et al; 2018). No entanto, trata-se de uma dependência, um problema de saúde pública, que merece cuidado, políticas públicas e acompanhamento contínuo por equipe multiprofissional.

Quando a gestante procura os serviços de saúde, muitas vezes omite a condição de dependente química por receio de ser repreendida e desaprovada pelas 
equipes de saúde (KASSADA et al., 2013, p. 470; BRASIL, 2012). A concepção de gênero e os papéis sociais esperados que a mulher desempenhe tais como mãe, esposa e zeladora da família, entram em choque com o consumo de drogas, ainda mais na gestação. A mulher passa a contrariar duplamente as normas sociais, primeiro pelo próprio uso, segundo porque compromete o desempenho dos papeis que nela são projetados (OLIVEIRA; PAIVA, 2007). E esse julgamento por parte de alguns profissionais contribui para que a mulher omita ou negue o uso, acarretando num maior grau de exposição à droga e aos agravos de saúde.

Os sentimentos, conceitos e valores socialmente construídos através do senso comum, direcionam para atendimentos carregados de preconceito, impactando no cuidado, na atenção às demandas, no seguimento do pré-natal e no vínculo (CORDEIRO, 2014; MARCOLINO et al; 2018). Esse senso comum caracteriza as pessoas usuárias de drogas como perigosas, criminosas, em situação de rua, gerando indagações e condutas profissionais diferenciadas (CORDEIRO, 2014).

O problema das drogas é uma questão de saúde pública que se relaciona com diversos fatores externos, mas que são vistos, geralmente, como problemas individuais que a qualquer momento podem ser sanados, basta ter vontade e persistência (OLIVIO; GRACZYK, 2011). Quando se trata do gênero, o peso que recai sobre as mulheres que fazem uso abusivo de drogas, gera olhares de incapacidade para realização dos cuidados de uma criança, como se elas estivessem sentenciadas e incapacitadas a desenvolver a maternidade pelo uso das drogas (SOBOTA, 2014; CAMARGO et al; 2018).

O estigma pela mulher gestante e dependente química e o não acompanhamento satisfatório nos serviços, pode acarretar diversas intercorrências de saúde, evoluindo muitas vezes para uma gestação de alto risco (MAIA; PEREIRA; MENEZES, 2015). No entanto, a capacitação insuficiente dos profissionais de saúde e a não oferta de suporte necessário na atenção primária a estas gestantes, são fatores que contribuem para que os hospitais sejam a única porta de acesso a estas usuárias e não raro, apenas no momento do parto (YABUUTI; BERNARDY, 2014).

A Rede Cegonha do Sistema Único de Saúde (SUS), que é o modelo de atenção voltado ao cuidado materno-infantil e que visa o planejamento reprodutivo, 
atenção à gestação, ao parto e ao puerpério, não apresenta nos fatores de risco o encaminhamento ao pré-natal de alto risco das mulheres que fazem uso abusivo de drogas (BRASIL, 2011). Entende-se que, por se tratar de drogas nocivas que interferem no desenvolvimento do bebê e na saúde da gestante, a abordagem precisa ser direcionada de modo a contemplar os riscos e as vulnerabilidades inerentes a esta situação, bem como um cuidado intersetorial (UNASUS, 2016).

Segundo pesquisa realizada pela Fundação Oswaldo Cruz (Fiocruz) em 2013, nos vinte e seis estados brasileiros, aproximadamente 370 mil pessoas utilizaram crack e um terço dessas não fizeram uso de preservativos em suas relações sexuais. Registra-se que $80 \%$ eram homens e $20 \%$ eram mulheres, das quais $10 \%$ estavam gestantes no momento da pesquisa (FIOCRUZ, 2013). Soma-se a este levantamento um estudo nacional com 394 gestantes, em que foi identificado que $18 \%$ das entrevistadas faziam uso abusivo de drogas lícitas e ilícitas durante a gestação, sendo que as mais utilizadas eram, respectivamente, o cigarro seguido do álcool, e a cocaína e seu derivados, seguido do crack e da maconha (KASSADA et al., 2013).

Salienta-se que as condições subjetivas e objetivas de vida dessas usuárias, tais como baixa escolaridade, multiparidade e exposição a riscos, impactam diretamente sobre os danos físicos e os desfechos sociais, como por exemplo, indo de intercorrências graves de saúde, rede de apoio fragilizada, laços familaires rompidos, à destituição do poder familiar (PORTO et al., 2018). Frente a esta realidade, as equipes de saúde necessitam compreender precisamente a problemática, para que estejam aptas na realização de um atendimento acolhedor e integral (BRASIL, 2012).

A intersetorialidade, a clínica ampliada e o cuidado compartilhado, devem estar alinhados no cotidiano das equipes, onde tanto a atenção primária, quanto a rede especializada psicossocial, assumam a corresponsabilização do cuidado (PETERS, et al; 2020). É exatamente durante a gestação que se encontram os facilitadores para a redução do uso, pois o desejo da maioria das mulheres dependentes químicas é não prejudicar o bebê, realizando os cuidados e permanecendo com ele após o nascimento (BRASIL, 2012). 
Desde 1994 utiliza-se a estratégia da Redução de Danos, com o intuito de ampliar as possibilidades de cuidado das pessoas que, em função do uso de drogas, tornam-se mais vulneráveis a outros agravos de saúde (SIQUEIRA, 2014; BRASIL, 2004). Trata-se de "um conjunto de estratégias de saúde pública voltada para a minimização das consequências causadas por práticas de riscos relacionadas ao uso das drogas" (SIQUEIRA, 2014, p. 67).

No entanto, os princípios e as diretrizes atuais das políticas públicas para o cuidado de usuários de álcool e outras drogas vêm criticando amplamente o oferecimento de intervenções consideradas padrões para o tratamento, como por exemplo, as internações, pois cada sujeito possui especificidades e singularidades que devem fazer parte do planejamento e das ações de cuidado (CORDEIRO, 2014). Neste sentido, não se deve abordar a problemática da gestação e do uso de drogas pelo viés moralizante e institucional, pois pode-se potencializar os riscos, agravos e vulnerabilidades. São as práticas humanizadas, acolhedoras e respeitosas que proporcionarão o vínculo e a permanência das mulheres nos serviços (PETERS, et al; 2020).

Entende-se que é complexa a temática abordada, sendo ainda mais acentuada quando o grupo em foco faz parte de uma população culturalmente estigmatizada, discriminada e com papeis sociais definidos, principalmente quando adotam um comportamento socialmente considerado desviante (OLIVEIRA; PAIVA, 2007). Enfatiza-se que a oferta de capacitações e espaços para discussão e reflexão sobre as práticas profissionais em saúde, o papel dos serviços, as potencialidades da rede de cuidados, são necessárias e essenciais para a qualidade da oferta de cuidados e aperfeiçoamento das equipes (SOBOTA, 2014). É através desse cuidado humanizado, respeitoso, compartilhado entre todos setores envolvidos que 0 acompanhamento, atendimento e o pré-natal serão de qualidade.

\section{CONCLUSÃO}


EDUCAÇÃO, SAÚDE

ETECNOLOGIA

26 A 28 DE OUTUBRO DE 2021

No contexto de gestação e uso de drogas, destaca-se a importância de realizar um atendimento respeitoso e acolhedor que vise um cuidado de corresponsabilização da rede intersetorial. Nessa realidade, a possibilidade de ser atendida e ouvida por uma equipe que possibilita um espaço facilitador de diálogo, de compreensão e de respeito aos problemas e dilemas que vivenciam, contribui para que a mulher crie vínculo e tenha permanência no acompanhamento pré-natal. Deste modo, salienta-se a necessidade de discussões, aperfeiçoamentos e educação permanente das equipes no atendimento desta demanda, oportunizando todo o suporte e tratamento adequado para um atendimento de qualidade.

\section{REFERÊNCIAS}

BRASIL. Ministério da Saúde. Secretaria de Atenção à Saúde. Departamento de Ações Programáticas Estratégicas. Álcool e redução de danos: uma abordagem inovadora para países em transição. 1. Ed; Brasília, Ministério da Saúde, 2004. p. 29-36.

Rede Cegonha. Ministério da Saúde. Brasília, DF, 2011.

.Ministério da Saúde. Secretaria de Atenção à Saúde. Departamento de Ações Programáticas Estratégicas. Gestação de alto risco: manual técnico, 5. Ed; Brasília, 2012, p. 11-24.

Portaria $\mathbf{n}^{0} \mathbf{1 . 0 2 0}$, de 29 de maio de 2013. Institui as diretrizes para a organização da Atenção à Saúde na Gestação de Alto Risco e define os critérios para a implantação e habilitação dos serviços de referência à Atenção à Saúde na Gestação de Alto Risco, incluída a Casa de Gestante, Bebê e Puérpera (CGBP), em conformidade com a Rede Cegonha.

BRITES, C. Dilemas na atuação com gestantes usuárias de drogas. In: Boletim Conexão Geraes $n^{\circ}$ 70/2015. 


\section{QUFN}

CAMARGO, PO. et al. O enfrentamento do estigma vivido por mulheres/mães usuárias de crack SMAD, Rev. Eletrônica Saúde Mental Álcool Drog. (Ed. port.), v.14, n. 4, Ribeirão Preto, out./dez. 2018.

\section{CARLINI, EA. et al. II levantamento domiciliar sobre o uso de drogas}

psicotrópicas no Brasil: estudo envolvendo as 108 maiores cidades do país 2005. São Paulo: CEBRID - Centro Brasileiro de Informação sobre Drogas Psicotrópicas: UNIFESP - Universidade Federal de São Paulo, 2006.

CORDEIRO, F. Representação social do uso de drogas, do usuário e do cuidado. In OLIVEIRA, Walter Ferreira de; CARNEIRO, Henrique (orgs.). Álcool e sociedade. Florianópolis: Departamento de Saúde Pública/UFSC, Unidade 2, 2014, p. 35-53.

FUNDAÇÃO OSWALDO CRUZ. Pesquisa nacional sobre o uso de crack e outras drogas, 2013.

KASSADA, SD. et al. Prevalência do uso de drogas de abuso por gestantes. Acta paul. enferm. São Paulo, v. 26, n. 5, p. 467-471, out. 2013.

MAIA, JA; PEREIRA, LA; MENEZES, FA. Consequências do uso de drogas na gravidez. In: Revista Enfermagem Contemporânea, Bahia, v. 4, n. 2, p. 121-128, jul./dez. 2015.

MARCOLINO, TQ. et al. Gestação e uso de substâncias psicoativas: qual é o cuidado em saúde desejado pelas mulheres? Cad. Saúde Colet., 2018, Rio de Janeiro, v. 26 , n. 3, p. 255-260.

OLIVEIRA, JF; PAIVA, MS. Vulnerabilidade de mulheres usuárias de drogas ao HIVIAIDS em uma perspectiva de gênero. Escola Anna Nery Revista de Enfermagem, Rio de Janeiro, 2007, v. 11, n. 04, p. 625-631. 


\section{QUFN}

OLIVIO, MC; GRACZYK, RC. Mulheres usuárias de crack e maternidade: breves considerações. In: Anais II Simpósio Gênero e Políticas Públicas, Universidade Estadual de Londrina, 18 e 19 de agosto, 2011.

PETERS, AA. et al. Gestantes em uso de substâncias psicoativas atendidas por enfermeiros na Atenção Primária à Saúde. SMAD, Rev. Eletrônica Saúde Mental Álcool Drog, v.16, n.2, Ribeirão Preto, abr./jun. 2020

PORTO, PN. et al. Fatores associados ao uso de álcool e drogas por mulheres gestantes, Revista Rene, Fortaleza, 2018, v.19, p. 1-7.

SIQUEIRA, D. Redução de danos no SUS. In BÜCHELE, Fátima; DIMENSTEIN, Magda (orgs.). Recursos e estratégias do cuidado, Florianópolis: Departamento de Saúde Pública/UFSC, 2014, Unidade 3.

SOBOTA, J. O cuidado destinado às gestantes usuárias de crack: as percepções da equipe do pré-natal de alto risco de uma unidade do sul do Brasil. Dissertação (mestrado profissional), Universidade Federal de Santa Catarina, 2014. p. 25- 68.

SOCCOL, KLS. et al. O cotidiano das relações familiares com indivíduo dependente químico. Revista Cogitare Enfermagem, Paraná, 2014, v. 19, n. 1, p.116-122.

UNIVERSIDADE ABERTA DO SUS. UNASUS/UFMA. Conceitos básicos sobre o uso abusivo e dependência de drogas. São Luíz: Maranhão, 2013, p. 1-14.

YABUUTI, PLK; BERNARDY, CCF. Perfil de gestantes usuárias de drogas atendidas em um centro de atenção psicossocial. Revista Baiana de Saúde Pública. Bahia, v. 38, n. 2, p. 344-356, abr./jun. 2014. 